\title{
Simple-Adaptive Link State Update Algorithm for QoS Routing ${ }^{\star}$
}

\author{
Seung-Hyuk Choi ${ }^{1}$, Myoung-Hee Jung ${ }^{1}$, Min Young Chung ${ }^{1, \star \star}$, \\ Mijeong Yang ${ }^{2}$, Taeil Kim², and Jaehyung Park ${ }^{3}$ \\ 1 School of Information and Communication Engineering, Sungkyunkwan University \\ 300, Chunchun-dong, Jangan-gu, Suwon, Kyunggi-do, 440-746, Korea \\ \{zealion, aodgl, mychung\}@ece.skku.ac.kr \\ 2 Broadband Converged Network Division, ETRI \\ 161, Gajeong-dong, Yuseong-gu, Daejeon, 305-700, Korea \\ \{mjyang, tikim\}@etri.re.kr \\ 3 Department of Computer Engineering, Chonnam National University \\ 300, Yongbong-dong, Buk-ku, Gwangju, 500-757, Korea \\ hyeoung@chonnam.ac.kr
}

\begin{abstract}
To guarantee Quality of Service, routers should automatically determine routing paths in order to satisfy service requirements efficiently, based on link state information as well as network topology. Link State Database (LSDB) in routers should be well managed in order to reflect the current state of all links effectively. However, there is a trade-off between the exact reflection of the current link status and its update cost. In this paper, a simple-adaptive LSU algorithm to adaptively control the generation of link state update messages is proposed and its performance is compared with those of four existing algorithms by intensive simulations.
\end{abstract}

\section{Introduction}

In general, in order to guarantee QoS, routers determine routing paths by considering link state information as well as network topology. Therefore, it is important that routers know link state information to calculate the routing paths, i.e., information in Link State Database (LSDB) resided in all routers should be well managed. To reflect the link status in the LSDB, routers transmit LSU messages to their neighbors. If routers generate Link State Update (LSU) messages inordinately, the router performance is reduced due to the processing of LSU messages [1. However, in the case that link state information is not updated appropriately, route setup requests may be rejected even though routes exist. This problem with QoS routing therefore can be characterized by the trade-off

\footnotetext{
* This study was partially supported by the Smart(Ubiquitous) Seoul Project titled "An Intelligent Convergence System of Urban Information for Smart(Ubiquitous) Cities" which was operated by the University of Seoul and funded by City of Seoul, Korea(South).

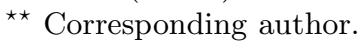


between the accuracy of link state information and the overhead incurred by exchanging this information [2].

Many algorithms exist for determining the instant of transmitting LSU messages, i.e., Period Based (PB), Threshold Based (TB), Equal Class Based (ECB), Unequal Class Based (UCB) LSU algorithm [3, 4], Dynamic Threshold Based (DTB) [5], and Second-moment Based (SB) 3] LSU algorithms. The existing LSU algorithm uses fixed value(s) in order to decide transmission of LSU messages, therefore they cannot effectively perform under different network topologies and traffic conditions. erfectly to network conditions.

\section{The Proposed Algorithm}

Since the existing algorithms use fixed value(s) in order to determine the transmission instant of LSU messages, they may perform ineffectively depending on varying network topology and traffic conditions. To overcome this disadvantage, a Simple Adaptive (SA) LSU algorithm is proposed, where parameter values adaptively change as the link state changes.

In the proposed algorithm, in order to determine whether to transmit LSU messages, routers observe available bandwidth and the number of serving connections on a link. For every request for a connection setup (or release), the available bandwidth increases (or decreases) by the corresponding request bandwidth, and the number of connections served increases (or decreases) by one. If LSU messages are flooded, all receiving routers update their LSDB as information contained in LSU messages.

Let $B_{n}$ be the available bandwidth value stored in the LSDB. In addition, let $\widetilde{B}(t)$ and $\widetilde{N}(t)$ be the current available bandwidth on links and the number of serving connections on a link, respectively, immediately prior to making the decision to transmit LSU messages. Routers transmit LSU messages to their neighbors if the following condition is satisfied.

$$
\begin{aligned}
\left|B_{n}-\widetilde{B}(t)\right| & \geq \frac{\widetilde{B}(t)}{\widetilde{N}(t)} \\
\Longleftrightarrow \frac{\left|\widetilde{U}(t)-U_{n}\right|}{1} & \geq \frac{C}{\widetilde{N}(t)}-\frac{\widetilde{U}(t)}{\widetilde{N}(t)},
\end{aligned}
$$

where $\widetilde{U}(t)$ denotes unavailable bandwidth immediately prior to making a decision to transmit LSU messages, $U_{n}$ indicates unavailable bandwidth stored in the LSDB, and $C$ expresses the total capacity of one link.

Consequently, in the SA LSU algorithm, if the variation in used bandwidth per service is equal to or larger than the mean available bandwidth per connection, expected at the instant of the next link state update, LSU messages are transmitted. Unlike the other algorithms, since the SA LSU algorithm only uses the control parameters about link state, it is able to effectively update the LSDB. 


\section{Performance Evaluation}

In order to evaluate the performance of the proposed algorithm, an MCI topology consisting of 18 nodes and 30 bidirectional T3 links (45 Mbps) is considered 3]. Each connection request is defined as $\left(s, d, b_{r e q}\right)$, where $s, d$, and $b_{r e q}$ denote source node, destination node, and request bandwidth, respectively. For each connection, $s$ and $d$ are different and randomly selected from 18 nodes. In addition, $b_{r e q}$ is uniformly chosen in (3Mbps, $\left.7 \mathrm{Mbps}\right)$, and it assumes that connection requests arrive as a Poisson process with the mean arrival rate $\lambda$ and holding duration of connections is determined by the exponential distribution with the average service rate $\mu$. Therefore, total offered traffic load is equal to $\lambda / \mu$.

As performance measures, the number of updates per unit time and blocking probability are defined. The number of updates per unit time is defined that the total number of LSU messages transmitted is divided by the total simulation time. The blocking probability of connection requests is defined as

$$
P_{\text {block }}=\frac{N_{\text {block }}}{N_{\text {total request }}}
$$

where $N_{\text {total request }}$ is the total number of connection requests and $N_{\text {block }}$ is the number of blocked connection requests.

In general, performance of the existing algorithms relies heavily on the used values of control parameters. For each of them, the specified value(s) are determined as the pseudo-optimal value(s) of parameter(s) if the blocking probability is close to that of the basic LSU algorithm in which LSU messages are generated whenever the available bandwidth varies. For the MCI network and $\mu=1 /$ sec., the pseudo-optimal values of control parameters considered for the the existing three algorithms are $t h=0.2$ for $\mathrm{TB}, R_{0}=0.1$ and $\Delta t h=0.5$ for DTB, and $t h=0.03$ for SB LSU algorithm. These pseudo-optimal values are used in order to evaluate the performance of the three existing algorithms.

For $\mu=1 /$ sec., the blocking probabilities of the four existing algorithms, and the proposed algorithm is presented in Fig. 1. The blocking probability of the basic LSU algorithm provides the least margin of those, because this algorithm makes an update of the status-changed information immediately when routers sense link status change. The blocking probability of the connection requests increases in proportion to the traffic load. Each existing LSU algorithm with the pseudo-optimal value(s) demonstrates a blocking probability which is analogous to that of the basic LSU algorithm. In addition, the blocking probability in the proposed LSU algorithm is similar to that of the basic LSU algorithm.

Fig. 2 presents the LSU rates varying traffic load. In the three existing LSU algorithms, the parameter value(s) are fixed.

On the other hand, in the SA LSU algorithm, the values used to determine LSU message transmissions are calculated considering network traffic conditions. Therefore, routers effectively transmit LSU messages and make the smaller number of updates than the existing LSU algorithms. 


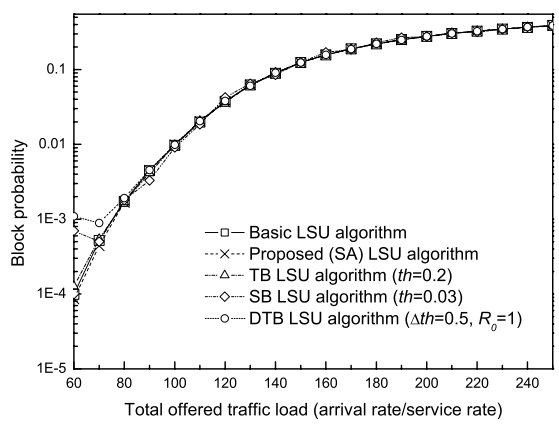

Fig. 1. Blocking probability

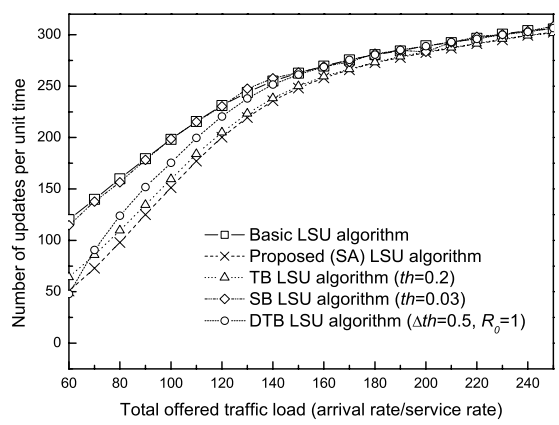

Fig. 2. LSU rate

\section{Conclusions}

In this paper, the SA LSU algorithm is proposed and its performance is evaluated by simulations. Existing LSU algorithms decide to transmit LSU messages by fixed parameter value(s). Therefore, the associated performance may degrade with varying network conditions. However, since the SA LSU algorithm decides whether to transmit or not LSU messages by parameters which are calculated from traffic conditions, the associated performance dose not depend on traffic conditions. In addition, in the proposed LSU algorithm, while the LSU messages are transmitted less than existing LSU algorithms, the blocking probability of connection requests is similar to that of existing LSU algorithms. Additionally, because the SA LSU algorithm requires simple computations, it may be easily implemented in QoS routers.

\section{References}

1. Apostolopoulos, G., Guerin, R., Kamat, s.: Implementation and Performance Measurements of QoS Routing Extensions to OSPF. IEEE INFOCOM 1999, Vol. 2., New York (1999) 680-688

2. Jia, Y., Nikolaidis, I., Gburzynski, P.: Multiple Path Routing in Networks with Inaccurate Link State Information. ICC 2001, Vol. 8., IEEE, Helsinki (2001) 25832587

3. Zhao, M., Zhu, H., Li, V.O.K., Ma, Z.: A Stability-Based Link State Updating Mechanism for QoS Routing. ICC 2005, Vol. 1., IEEE, Seoul (2005) 33-37

4. Ma, Z., Zhang, P., Kantola, R.: Influence of Link State Updating on the Performance and Cost of QoS Routing in an Intranet. 2001 IEEE Workshop on High Performance Switching and Routing, Dallas (2001) 375-379

5. Ariza, A., Casilari, E., Sandoval, F.: QoS routing with adaptive updating of link states. Electronics Letters, Vol. 37., IEEE, (2001) 604-606 